\title{
The Research on Surface Restructure Method of Discrete Data Based on Multi-cores Environment
}

\author{
Huijiang Zheng ${ }^{1,3}$, Jing Zhang ${ }^{2}$, Baihui Ding ${ }^{1}$, Gaiyun $\mathrm{He}^{1}$ \\ ${ }^{1}$ Tianjin Key Lab. for Advanced Manufacturing Tech. and Equipment, Tianjin University, Tianjin, \\ 300072, China; \\ ${ }^{2}$ School of Control \& Mechanical Engineering, Tianjin Chengjian University, Tianjin, 300384, China; \\ ${ }^{3}$ School of Mechanical \& Aerospace Engineering, Queen's University Belfast, Belfast BT9 5AH, UK.
}

Keywords: Multi-cores Environment, Discrete Data, Surface Restructure, Parallel Computation.

\begin{abstract}
Surface restructure for triangulation discrete data was the preconditions for discrete data to be widely applied in modern manufacturing. Due to using multi-core processors and connected graph traversal access algorithm, put forward a new method of surface restructure for discrete data based on parallel undirected graph. This method played the characteristics of multi-core environment, used inherent storage relation between triangulation data and the independent character of traversal for undirected graph. In this method, build the relation model between triangles based on undirected graph structure, optimize the method for processing concurrent access, and improve the efficiency of calculation. The result of examples showed that the method would have concurrent access a better performance than the existing algorithms, could significantly improve the efficiency of surface restructure for discrete data and enhance the stability of the calculation method.
\end{abstract}

\section{Introduction}

Multi-core processors are integrated multiple fully functional core in the same chip, and by the whole chip as the unified structure provide services to system [1,2]. In a multi-core processor, often integrate with multiple single thread processing cores or multiple simultaneous multi thread processor cores. The whole processor number of executing threads at the same time is a single processor several times. It will improve the efficiency of the processor for processing data [3].

In the field of modern manufacturing, we often need process trajectory calculation, detection point distribution or the related data analysis and mining to model the parts of a surface or some surface [4-6]. While using CAD software derived model data of parts, especially to some part models with complex surface, a large number of spatial discrete triangle datum is formed by the parts surface triangulation. Then data storage and data transfer between the used environments are carried out.

Due to triangulate datum no longer explicitly contain the original surface configuration information, the subsequent processing of the parts surface feature recognition or the analysis according to the surface characteristics of the parts is difficult. In previous methods, the surfaces were restructured with boundary extraction, boundary loop tracking, and the list of boundary ring [4]. This method will require a longer time for the calculation to surface reconstruction of the parts with high-precision complex surface. Therefore the subsequent processing of triangle discrete data is inconvenient.

In this paper, using the multi-core architecture and undirected graph traversal access algorithm, put forward a new method of surface restructure for discrete data based on parallel undirected graph by solving the problems of parallel traversal access conflicts and surface fragments of merge.

\section{A new method of surface restructure based on parallel undirected graph structure}

\section{Surface model of the parts based on undirected graph structure}

In the existing data structure, graph can be written as a triple $\mathrm{H}$. Then the expression for $\mathrm{H}$ is [7]: 


$$
H=<V(H), E(H), \varphi(H)>
$$

Among them, $V(H)$ is a collection of node elements in $H$. $E(H)$ is a collection of edges in $H$. $\varphi(H): E \rightarrow V \times V$ is known as copula function. Fig.1 shows if every element in $E(H)$ is directionless, $H$ is an undirected graph. Starting from an arbitrary element in $V(H)$, access to the rest of the node elements in $H$ along $E(H)$, and each node has been visited only once. This process is known as traverse of graphs [7].

Through the existing effective algorithms, the basic topological relations can be established between the triangle discrete data [4]. Using the existing basic topological relationship, combining with the basic concepts of graph, with the alone triangular face as node, with the adjacent relations of the alone triangular face and the other as edges, can construct undirected graph model which contains all triangles. In this model, the relationship between the vertex elements is stored using the structure of class adjacency list. That is to say, a vertex element as a head node and all of its adjacent vertices are put in the same adjacent list. Every vertex will correspond to a chain.

In accordance with the rules of spatial geometric triangulation [4]: each of the triangles and adjacent triangles have only two common points, adjacent triangles have only one public side. Then each triangle has three adjacent triangles. So in the undirected graph model above, each vertex element associated list number is three.

The model above can effectively cover all the surface of the whole parts, but the actual work often need to deal with one or several surface, which need to construct corresponding surface set with part of triangles, namely, to realize the reconstruction of surface faces.

The realization of surface face reconstruction effectively in discrete data, there are two core problems to solve. First, establish the accurate and valid logical dependency relations between triangular face and surface face. Second, accurately find the boundary of two adjacent surface faces.

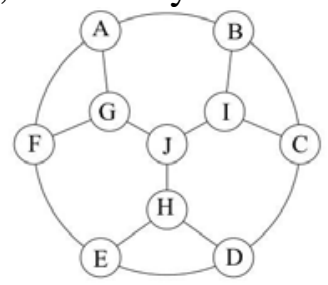

Fig.1 Diagrammatic sketch of undirected graph $H$
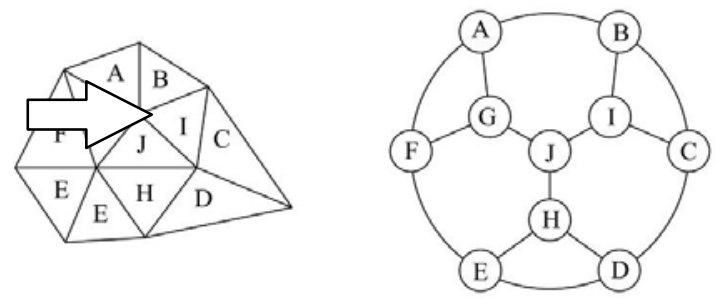

Fig.2 Diagrammatic sketch of graph $H$

If the definition of a surface is $H$, each triangle in the inner of $H$ will be adjacent at least with one triangle in $H$. Obviously, at least one path of $H$ makes any two vertex connectivity, and $H$ is a connected graph. Fig. 2 shows the structure model of it. The graph structure model of part surface can also be looked as a number of connected graphs sets which are similar to $H$. The use of $H$ internal connectivity to find all the triangles it contains is a basic idea to solve the first problem.

If the surface face which is adjacent with surface face $H$ is defined as $H^{\prime}, H^{\prime}$ also should be a connected graph. Because of $H$ and $H^{\prime}$ are composed of triangles, their boundary will be common boundary set of several adjacent triangles in two surface faces.

Threshold $\lambda$ can be selected according to the accuracy of triangulation of surface discrete data.

In the discrete data space, let two arbitrary adjacent triangles be a and $b$, their unit normal vectors are respectively $\boldsymbol{n}_{a}$ and $\boldsymbol{n}_{b}$. The angle between $\boldsymbol{n}_{a}$ and $\boldsymbol{n}_{b}$ is $\theta$. While the expression for $\theta$ is

$$
\theta=\arccos \left(\boldsymbol{n}_{a} \cdot \boldsymbol{n}_{b}\right)
$$


If $\theta<\lambda$, a and $\mathrm{b}$ belong to the same surface patch. Conversely, $\mathrm{a}$ and $\mathrm{b}$ belong to the different surface patch, and adjacent side of $a$ and $b$ is the boundary line of adjacent surface patches [4] . If this time has determined the surface patch which $a$ and $b$ are belong to, we could established the adjacent relationship between the two surface patches. After establish the relationship between surface patch and all triangles in it, the adjacent relationship between surface patches could be established.

From the above analysis, it is not difficult to the following two conclusions:

1. For a connected graph $H$, starting from an arbitrary vertex inside of it, traversal access to all the vertices can be completed. The different selection of the initial vertex only affects the final access sequence.

2. In addition to the triangles in the two adjacent surface patches boundary, traversal access to the vertexes in connected graph $H$ is independent of the vertexes in the other graphs.

\section{Surface restructure method based on parallel computation}

If the accuracy of parts surface triangulation is higher, the number of triangle is bigger. Single thread traversal access time will be rapid growth. The above analysis concluded in multi-core computer, the efficiency of surface reconstruction can be improved, through constructing traversal access algorithm based on parallel undirected graph, by simultaneous traversal access for multiple connected graphs contains in discrete data.

In the parallel traversal specific algorithm, first needs to solve the problem of concurrent access competition [8]. To solve this problem, before threads access shared data in the triangle C, lock share datum with lock in operating system [8], in order to prohibit the data to be accessible to other threads. But too much lock processing will reduce the efficiency of parallel access [8]. In order to further reduce the probability of concurrent access competition, according to the internal correlation of storage order of triangulation data [4], the triangle datum are divided into several average sub list to access, according to the number of calculation cores. Then assign to the specified calculation thread. In the process of parallel traversal the initial triangles each thread selected come from the same access list. That can effectively reduce the probability of concurrent access competition, and can make the parallel traversal access code efficiently to be reused. Parallel access specific algorithm can be achieved through process shown in Fig.3. It shows the process of the thread process 2 to $\mathrm{n}$ is the same as thread 1. In order to avoid over division to connected graph, it is necessary to merge and calculate sub surface patches, according to the actual situation of parts surface in space geometry, after all the process complete traversal access to triangle faces.

\section{The examples and performance analysis of algorithm}

Three kinds of the surface structure design, which were shown in Fig.4, were finished by 3D CAD software. The models were saved as triangulation discrete data. For each one of models, three kinds of triangulation discrete data with different data size were saved by adjusting the parameters. The serial undirected graph algorithm and the parallel undirected graph algorithm were used to reconstruct surfaces of models. Computing environment is as follows: OS is Windows 7 64bit SP1; function lib of OpenGL2.0; and memory capacity is 8GB; CPU is i7 2.7GHz.

The result of computing is shown in Table 1. The computation time of two algorithms were gotten from the mean time of 5 times computation for different data size. The standard deviation was shown in Table 1. Examples of the calculation results show that for all kinds of models, the efficiency of the surface reconstruction based on undirected graph structure is better than the serial graph algorithm. The Efficiency ratio is up to 1.78 in dual-core mode. 


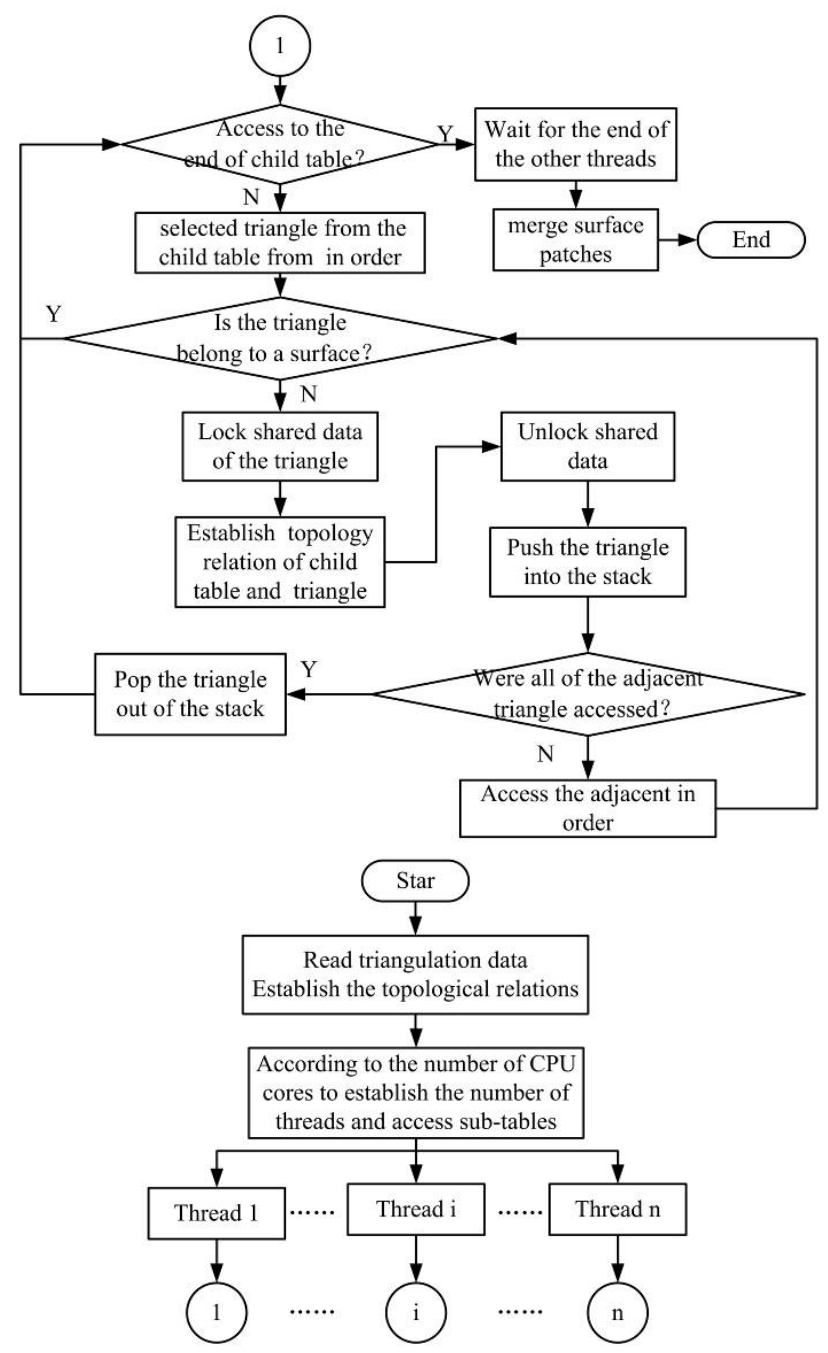

Fig.3 Flow chart of parallel traversal algorithm

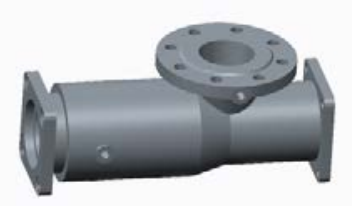

(a) Pump Body

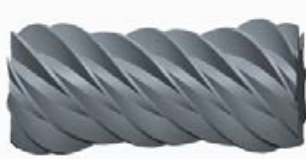

(b) Worm

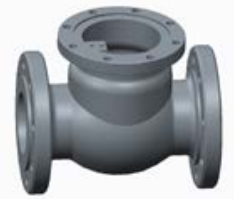

(c) Valve Body

Fig 4 The models used in testing

Table 1 The Results of Surface Reconstruction of Discrete data

\begin{tabular}{|c|c|c|c|c|c|c|c|}
\hline $\begin{array}{l}\text { the models } \\
\text { of discrete } \\
\text { data }\end{array}$ & $\begin{array}{l}\text { the size of } \\
\text { files(Byte) }\end{array}$ & $\begin{array}{l}\text { the amount } \\
\text { of triangles }\end{array}$ & $\begin{array}{l}\text { the amount } \\
\text { of surfaces }\end{array}$ & $\begin{array}{l}\text { the serial } \\
\text { undirected graph } \\
\text { algorithm (ms) }\end{array}$ & $\begin{array}{l}\text { the standard deviation } \\
\text { of time (serial } \\
\text { undirected graph } \\
\text { algorithm) (ms) }\end{array}$ & $\begin{array}{l}\text { the parallel } \\
\text { undirected graph } \\
\text { algorithm (ms) }\end{array}$ & $\begin{array}{l}\text { the standard deviation } \\
\text { of time (parallel } \\
\text { undirected graph } \\
\text { algorithm) (ms) }\end{array}$ \\
\hline $\begin{array}{l}\text { Pump } \\
\text { Body } 1\end{array}$ & $59,899,296$ & 231556 & 112 & 244.42 & 1.41 & 146.19 & 1.09 \\
\hline $\begin{array}{l}\text { Pump } \\
\text { Body } 2\end{array}$ & $119,733,571$ & 462872 & 112 & 512.96 & 3.24 & 288.79 & 1.38 \\
\hline $\begin{array}{l}\text { Pump } \\
\text { Body } 3\end{array}$ & $227,135,751$ & 932320 & 112 & 1045.18 & 6.48 & 588.00 & 3.19 \\
\hline Worm 1 & $57,403,826$ & 223396 & 18 & 241.91 & 0.97 & 144.66 & 0.54 \\
\hline Worm 2 & $114,939,643$ & 447310 & 18 & 485.58 & 2.17 & 290.22 & 1.94 \\
\hline Worm 3 & $229,634,557$ & 893652 & 18 & 1024.41 & 3.18 & 601.94 & 2.59 \\
\hline $\begin{array}{l}\text { Valve } \\
\text { Body } 1\end{array}$ & $57,935,585$ & 222532 & 214 & 245.46 & 2.99 & 149.24 & 1.90 \\
\hline $\begin{array}{l}\text { Valve } \\
\text { Body } 2\end{array}$ & $115,856,535$ & 445012 & 214 & 493.82 & 3.01 & 300.31 & 2.15 \\
\hline $\begin{array}{l}\text { Valve } \\
\text { Body } 3\end{array}$ & $231,546,864$ & 889392 & 214 & 1029.95 & 4.68 & 640.95 & 3.13 \\
\hline
\end{tabular}


In the large amount of data processing, the serial undirected graph algorithm and the parallel undirected graph algorithm exhibited good computational efficiency. It can been found from Table 1 that the increase of working time of undirected graph algorithm was linear relationship with the increase of data size. The standard deviations of parallel undirected graph algorithm were shorter than serial undirected graph algorithm in the same size of data size. It means that the parallel undirected graph algorithm had a very good stability in the large amount of data processing.

\section{Conclusion}

A method of surface restructure of triangulation discrete data was proposed by using the multi-core computing architecture. The method, which combined the advantages of a connected graph traversal, turned the surface reconstruction problem of discrete data into the problem of parallel undirected graph traversal to explore all of its connected graph. The surface reconstruction method using parallel undirected graph structure took full ad-vantage of multi-core environment and optimized the performance of concurrent access processing in parallel computing. The examples showed that the method could effectively deal with different data volume of discrete data and reflected better efficiency and stability in large amount of data processing, serial algorithm reflects better efficiency and stability, relatively to the serial algorithm.

\section{Acknowledgements}

This work was financially supported by the Project "The Research on the Method of Getting Manufacturing Quality Features for Complex Curved Surfaces Based on Spatial Data Mining” supported by National Natural Science Foundation of China (No. 51105271), the State Scholarship Fund supported by the China Scholarship Council (No.20140625508), Natural Science Foundation of Tianjin (No. 11JCYBJC06200). Huijiang Zheng is the correspondent of this paper.

\section{References}

[1] Sutter H, Larus J. Software and the Concurency Revolution [J]. Queue: Multiprocessors Que, 2005, 3(7):54-62.

[2] Banikazemi M, Poff D, Abali B. Pam: A Novel Performance Power Aaware Meta-Scheduler for Multi-core Systems[C]// Proccedings of the 2008 ACM/IEEE Conference on Supercomputing. Piscaraway: IEEE Press, 2008:24-36.

[3] Chen Yongheng, Zuo Xianglin. Parallel Two-Way Enumeration Join Based on Multi-cores Environment [J]. Journal of Jilin University (Science Edition), 2014,52(1):59-64.

[4] Zheng Huijiang. Research on the Key Technology of the Topological Feature Reconstruction and Sampling Point Distribution in OMV [D]. Tianjin: Tianjin University, 2010.

[5] X Pan, K Chen, D Chen. Development of Rapid Prototyping Slicing Software Based on STL Model[C]//Jiang-Liang Hou etcl. Proceedings of the 2014 IEEE 18th International Conference on Computer Supported Cooperative Work in Design, Taiwan, 2014.5.21-23. Taiwan: IEEE, 2014: 191-195.

[6] Zhu Hu, Yang Xiaoguang. A Study on the Interference Detection and Correction in 5-Axis CNC Incremental Forming [J]. Journal of Mechanical Engineering, 2014, 50(7):168-174.

[7] W.T.Tutte. Graph Theory [M] . Cambridge: Cambridge University Press, 2004.

[8] Zhou Weiming. Multicore computing and programming [M]. Wuhan: Huazhong University of Science and Technology Press, 2009 